\title{
Cenplesto
}

\section{Redes de Interação na Ciência: mapeamento de comunidades de autores}

\author{
Roberto Mario Lovón Canchumani \\ Doutor; Universidade Federal do Rio de Janeiro, Rio de Janeiro, RJ, Brasil; \\ rlovon@gmail.com
}

\begin{abstract}
Resumo: Este artigo apresenta os resultados de uma proposta de identificação de comunidades de autores realizado na Universidade Federal do Rio de Janeiro (UFRJ). O objetivo foi identificar a tendência de agrupamento de autores da instituição ao longo do tempo, assim como também observar características mais específicas das principais comunidades identificadas. $O$ trabalho investigou 44.233 registros recuperados da base institucional SIGMA.UFRJ, de artigos publicados em periódicos no período de 2001 a 2012. Os dados foram organizados e analisados com auxílio dos softwares Excel e Gephi. As métricas de grau e modularidade/transitividade mostraram que, ao longo do período estudado, novas colaborações entre os autores da UFRJ foram estabelecidas, formando novos agrupamentos/comunidades de autores. As maiores comunidades de autores identificadas foram detalhadas, revelando suas principais especificações.
\end{abstract}

Palavras-chave: Comunidades de Autores. Redes de Colaboração. Coautoria.

\section{Introdução}

O desenvolvimento de pesquisas no campo de redes complexas tem apresentado, nas últimas décadas, significativos avanços na descrição e análise dos traços topológicos e dinâmicos de redes de tipo social, biológica ou tecnológica (BARABÁSI et al., 2002). Nesse cenário, um dos aspectos que tem concentrado particular atenção tem sido a existência de subconjuntos de nós fortemente entrelaçados, às vezes conectados e outras vezes desconectados da rede. Neste último caso, nos deparamos com agrupamentos ou comunidades (clusters) que se encontram ligadas ou não à rede (FORTUNATO, 2010).

Essas comunidades cumprem papel relevante nas propriedades de estruturas complexas. Identificar e analisar a natureza das comunidades aí ocultas é uma importante tarefa para revelar a organização informal e a natureza dos fluxos de informação que acontecem dentro desses sistemas complexos 


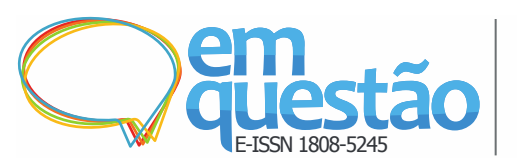

Redes de Interação na Ciência: mapeamento de

(PALLA et al., 2005). É importante destacar que tais comunidades são compostas por diferentes subconjuntos de nós, os quais se caracterizam por estarem densamente ligados mais entre si do que em relação ao resto da rede. A identificação dessas comunidades facilita a compreensão das redes.

Considerando que a comunidade cientifica atual se estrutura cada vez mais em um sistema integrado, cooperativo ou de rede, torna-se relevante identificar a natureza, a intensidade e as características específicas das interações entre seus membros (RIVELLINI; RIZZI; ZACCARIN, 2006; RODRIGUEZ, 1995).

Quando realizadas no contexto dos estudos da Informetria, essas análises buscam a representação dos laços colaborativos a partir das informações contidas na produção científica, mais frequentemente a partir da comunicação em formato de artigos em periódicos. Nesse âmbito, algumas abordagens têm permitido identificar comunidades científicas, nas quais se adotam funções específicas para descrever características dos grupos a serem determinados (CANCHUMANI; LETA; FIGUEIREDO, 2017; BARBOSA et al., 2011; PERIANES-RODRIGUEZ; OLMEDA-GÓMEZ; MOYA-ANEGON, 2010).

Este trabalho apresenta os resultados de uma proposta de mapeamento de comunidades de autores realizado na Universidade Federal do Rio de Janeiro (UFRJ). O objetivo do estudo foi identificar a tendência de agrupamento de autores da instituição ao longo do tempo, assim como também observar características mais específicas das principais comunidades de autores identificadas.

Cabe mencionar que uma primeira versão deste estudo foi apresentada no $6^{\circ}$ Encontro Brasileiro de Bibliometria e Cientometria (6 $6^{\circ}$ EBBC) (CANCHUMANI, 2018), realizado na cidade do Rio de Janeiro em julho de 2018. O presente artigo é um desdobramento desse trabalho, sendo, portanto, uma versão mais abrangente e detalhada dos resultados apresentados no $6^{\circ}$ EBBC. 


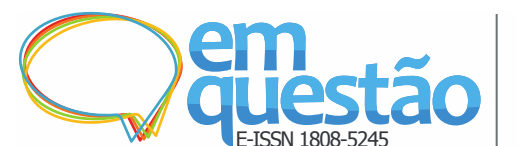

Redes de Interação na Ciência: mapeamento de comunidades de autores

Roberto Mario Lovón Canchumani

\section{Metodologia}

Foi realizado um levantamento da produção bibliográfica, envolvendo artigos publicados em periódicos no período de 2001 a 2012, na base de dados institucional SIGMA.UFRJ. O SIGMA.UFRJ foi criado em 1998 com a finalidade de ser um ambiente virtual e interativo de representação das atividades de ensino, de pesquisa e de extensão realizadas na UFRJ, construído sobre uma base de dados corporativa, na qual eram correntemente registradas essas atividades e evidenciadas suas múltiplas inter-relações.

Ressalta-se que até o ano de 2012, as informações da base SIGMA.UFRJ foram utilizadas nos processos de avaliação dos programas de pós-graduação da instituição, já que a coleta de dados era realizada nessa base, para posteriormente ser exportada para a Coleta CAPES. Em 2013, por decisões da Pró-Reitoria de Pós-Graduação da UFRJ, essa coleta deixou de ser realizada para fins avaliativos. A partir de então, a base se encontra inativa. Daí porque o período de análise se estende até 2012, último ano em que as informações da base, como já mencionado, foram atualizadas e utilizadas nos processos de avaliação dos programas de pós-graduação da UFRJ. Convém ressaltar também que essa base informacional ainda não tinha sido utilizada/explorada para estudos desta natureza.

A busca na mencionada base considerou os seguintes critérios: Produção Intelectual $\Rightarrow$ artigos completos publicados em periódicos $\Rightarrow$ autores. A partir desses critérios foi possível extrair 44.233 registros da produção da UFRJ de artigos publicados em periódicos no período de 2001 a 2012. Os registros com suas informações foram exportados para matrizes multidimensionais, um processo que usou ferramentas computacionais, como software para cruzamento de dados (Excel) e geração/visualização de redes/mapas (Gephi).

A interação entre autores foi analisada a partir das informações de coautoria e as métricas de grau e modularidade/transitividade. 


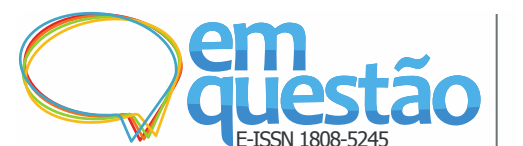

Redes de Interação na Ciência: mapeamento de comunidades de autores

Roberto Mario Lovón Canchumani

\section{Resultados}

A população identificada na produção acadêmica no período de 2001 a 2012 é constituída por 28.248 autores com vínculo formal com a UFRJ, responsáveis pelos 44.233 artigos publicados no período. A Figura 1 mostra o grafo que representa o total de autores $(n=28.248)$. Cada ponto do grafo representa um autor. Uma aresta na cor azul indica pelo menos uma publicação realizada em colaboração entre dois pesquisadores.

Figura 1 - Mapa de interação dos autores com vínculo formal com a UFRJ, de artigos publicados em periódicos no período 2001-2012

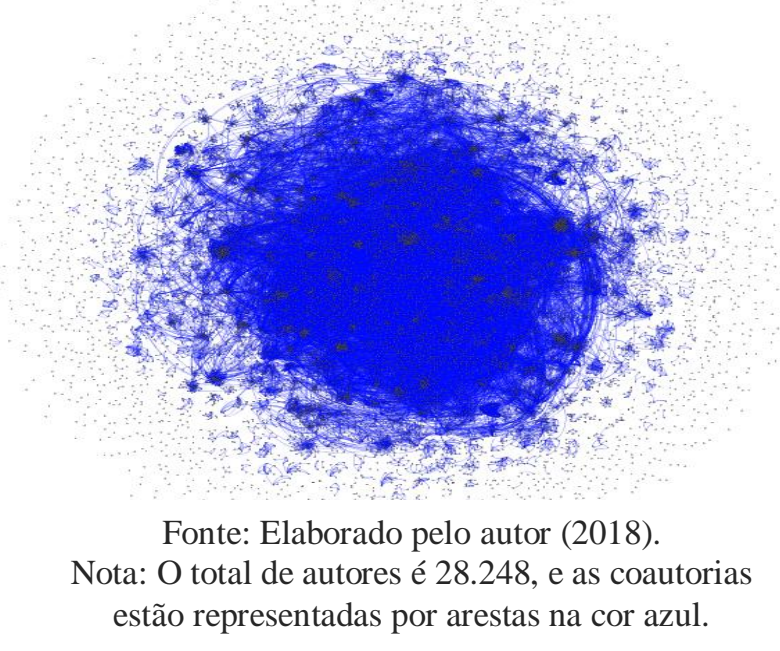

Os nós que aparecem visualmente isolados, mais externamente no grafo, correspondem a autores de artigos sem colaboração ou subconjunto de autores (geralmente formado por poucos pesquisadores) desligados da grande rede interligada.

Nas redes de interação de coautoria, em média, cada autor da UFRJ mantém colaboração com 6,73 pesquisadores. Observa-se que no grafo existem vários subgrupos, ou seja, conjuntos/agrupamentos de nós bem próximos uns dos outros, de autores que formam comunidades (clusters). Podemos notar, visualmente, que autores desses subgrupos colaboram mais entre si.

Um olhar por períodos (triênios), Figura 2, mostra que ao longo dos triênios a densidade do grafo aumenta. Essa simples representação visual possibilita observar o incremento da atividade colaborativa na UFRJ ao longo do tempo. 


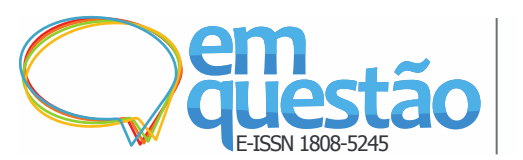

Redes de Interação na Ciência: mapeamento de comunidades de autores

Roberto Mario Lovón Canchumani

Figura 2 - Mapa de interação dos autores com vínculo formal com a UFRJ, de artigos publicados em periódicos por triênios

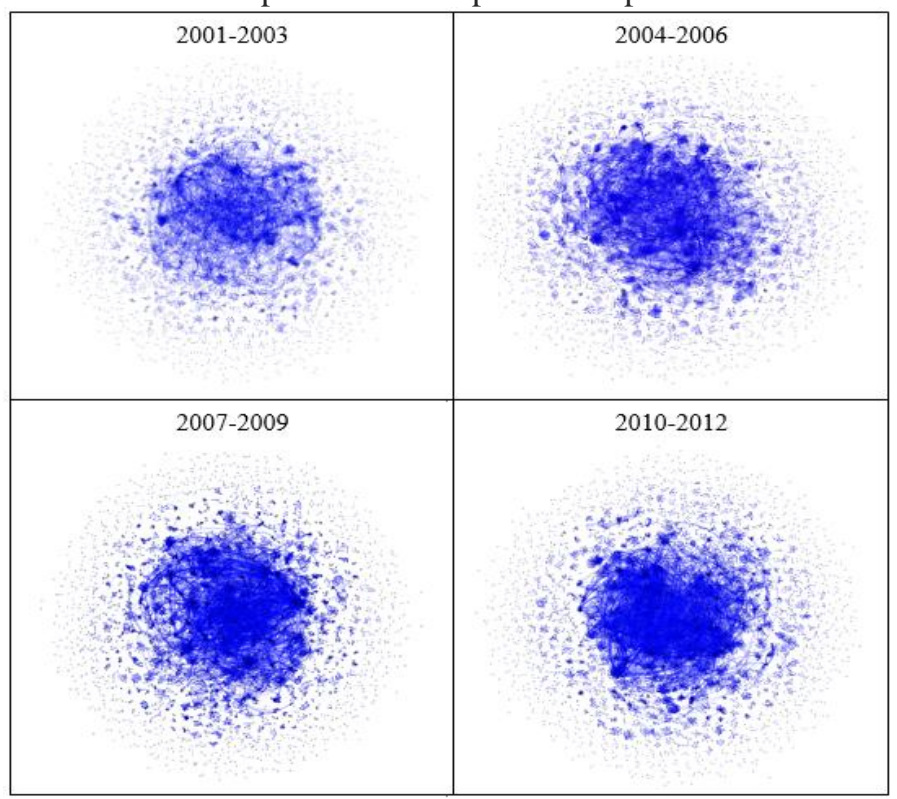

Fonte: Elaborado pelo autor (2018).

Nota: O total de autores é 28.248, distribuídos por triênios, e as coautorias estão representadas por arestas na cor azul.

Verificou-se que no triênio 2001-2003, o grau médio foi de 4,33, ou seja, cada autor colaborou, em média, com pouco mais de quatro autores, e a transitividade, isto é, a tendência que os autores têm de se agruparem (coeficiente de clustering) foi de 0,76. Em 2004-2006, o grau médio foi de 4,87 e o coeficiente de clustering foi de 0,77. No período seguinte, 2007-2009, o grau médio foi de 5,12 e a transitividade foi de 0,78. Já o último triênio apresentou grau médio de 5,57 e transitividade de 0,79.

Essas medidas indicam que, ao longo dos períodos, novas colaborações foram estabelecidas formando novos agrupamentos/comunidades. Em outras palavras, ao logo dos triênios, a tendência de todos os nós (autores) se agruparem aumenta.

A fim de verificar a rede de autores com participação mais intensa em termos de colaboração na instituição ao longo do tempo, identificamos a formação de diferentes subgrupos de pesquisadores que formam comunidades (clusters) para cada triênio, focalizando nossa atenção na maior comunidade de cada um desses períodos. 


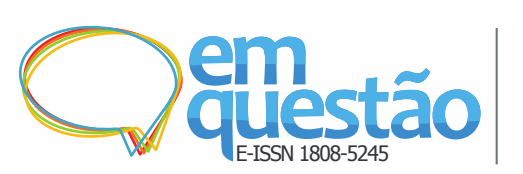

Redes de Interação na Ciência: mapeamento de comunidades de autores

Roberto Mario Lovón Canchumani

\subsection{Comunidades de autores no triênio 2001-2003}

A rede de coautoria da UFRJ, no período 2001-2003, é composta por 5.695 autores. A identificação de agrupamentos dessa população, aplicando o algoritmo Blondel et al. (2008), encontrou um total de 40 comunidades, envolvendo 3.736 nós (autores), identificadas na Figura 3-A através das cores.

Os nós (autores) estabeleceram entre si 11.304 relações (colaborações), representadas na rede por arestas. O grau médio foi de 6,05 , ou seja, em média, estes autores participaram de publicações com pouco mais de seis autores e a transitividade (coeficiente de clustering) de 0,76.

A maior comunidade de autores identificada no período de 2001-2003 é constituída por 236 autores (o que representa 7,04\% do total de autores das 40 comunidades) e, 689 relacionamentos, arestas (Figura 3-B). O grau médio é de 5,24, ou seja, cada autor colabora, em média, com mais de cinco pesquisadores. O coeficiente de transitividade apresentado é de 0,79.

Figura 3 - (A) Grafo das comunidades de autores da UFRJ de publicações em periódicos identificadas no período 2001-2003. (B) Grafo da maior comunidade de autores de artigos em periódicos da UFRJ no período 2001-2003

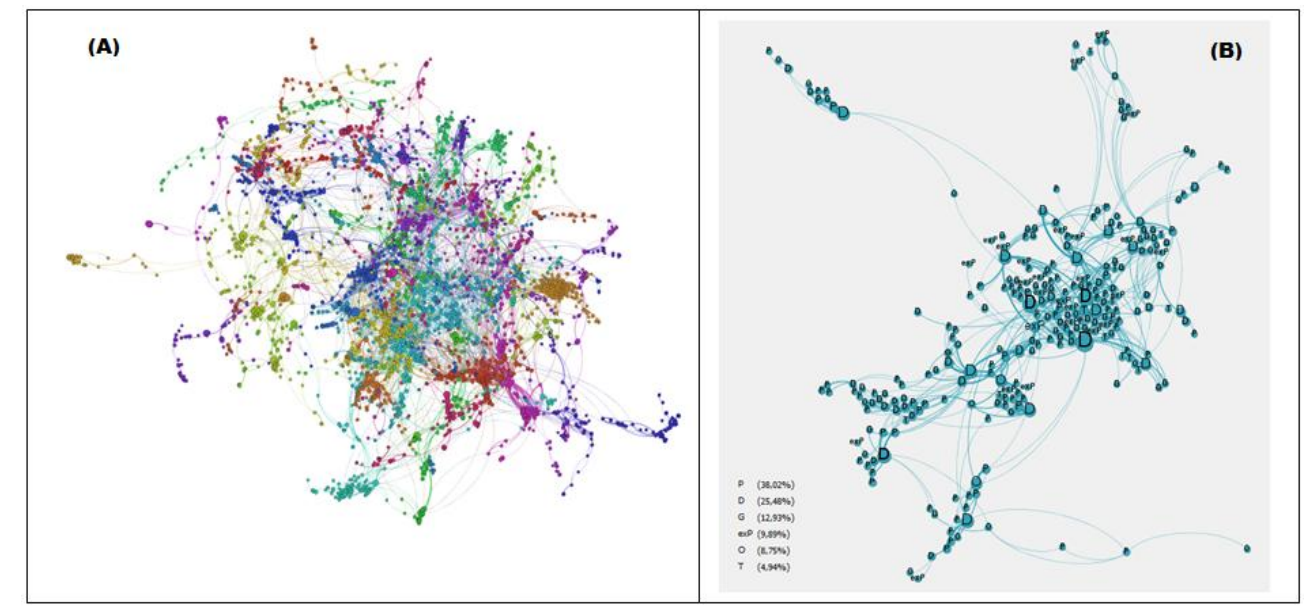

Fonte: Elaborado pelo autor (2018).

A classificação de autores dessa comunidade por tipo de vínculo mostra que a maioria é composta por alunos de pós-graduação - P $(38,02 \%)$, seguida por docentes - D (25,48\%) e alunos de graduação - G (12,93\%). Alunos egressos da pós-graduação (exP) representam 9,89\% e os autores com outros tipos de 


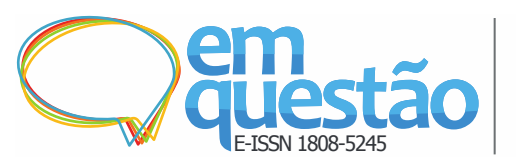

Redes de Interação na Ciência: mapeamento de comunidades de autores

Roberto Mario Lovón Canchumani

vínculo (O) 8,75\%, podendo ser professor egresso, técnico egresso ou egresso da graduação. Já os técnicos (T) representam 4,94\%.

Do total de autores dessa comunidade, identificamos os 20 que apresentam as maiores medidas de centralidade de grau, ou seja, são autores que mais interagem, tem maior número de colaborações (Tabela 1).

Verifica-se que dos 20 autores identificados, 16 são docentes. Os outros quatro autores têm os seguintes tipos de vínculo: egresso da pós-graduação (exP), aluno de pós-graduação $(\mathrm{P})$, técnico $(\mathrm{T})$ e um que possui outros tipos de vínculo $(\mathrm{O})$.

No que se refere às áreas dos autores, verificamos que dez deles atuam na grande área de Ciências Biológicas, dois nas Ciências da Saúde e um nas Ciências Humanas. Os outros autores identificados atuam em duas áreas do conhecimento, assim distribuídos: quatro deles em Ciências Biológicas e Ciências da Saúde; dois em Ciências Agrárias e Ciências da Saúde e; um autor em Ciências Biológicas e Ciências Humanas.

Tabela 1 - Vínculo e grande área dos autores com maiores medidas de centralidade de grau da maior comunidade identificada das publicações da UFRJ em 2001-2003

\begin{tabular}{|c|c|c|c|}
\hline Id & Vinculo* & Grau & Áreas de Atuação \\
\hline 330 & $\mathrm{D}$ & 46 & Ciências Biológicas; Ciências da Saúde \\
\hline 2139 & $\mathrm{D}$ & 34 & Ciências Biológicas, Ciências da Saúde \\
\hline 342 & $\mathrm{D}$ & 26 & Ciências Biológicas \\
\hline 339 & exP & 24 & Ciências Biológicas, Ciências da Saúde \\
\hline 343 & $\mathrm{O}$ & 20 & Ciências Biológicas \\
\hline 344 & $\mathrm{D}$ & 19 & Ciências Biológicas \\
\hline 2140 & $\mathrm{D}$ & 19 & Ciências da Saúde \\
\hline 268 & $\mathrm{D}$ & 17 & Ciências Biológicas \\
\hline 406 & $\mathrm{D}$ & 16 & Ciências Biológicas \\
\hline 2042 & $\mathrm{D}$ & 15 & Ciências Agrárias, Ciências da Saúde \\
\hline 2320 & $\mathrm{D}$ & 15 & Ciências da Saúde, Ciências Biológicas \\
\hline 445 & $\mathrm{D}$ & 14 & Ciências Biológicas \\
\hline 540 & $\mathrm{D}$ & 14 & Ciências Biológicas \\
\hline 2156 & $\mathrm{~T}$ & 14 & Ciências da Saúde \\
\hline 223 & $\mathrm{D}$ & 13 & Ciências Biológicas \\
\hline 412 & $\mathrm{D}$ & 12 & Ciências Biológicas, Ciências Humanas \\
\hline 1924 & $\mathrm{D}$ & 12 & Ciências da Saúde, Ciências Agrárias \\
\hline 338 & $\mathrm{P}$ & 11 & Ciências Humanas \\
\hline 341 & $\mathrm{D}$ & 11 & Ciências Biológicas \\
\hline 541 & $\mathrm{D}$ & 11 & Ciências Biológicas \\
\hline
\end{tabular}

Fonte: Elaborado pelo autor (2018).

* Tipos de Vínculo: D (Docente); P (Discente de Pós-graduação); exP (Egresso Discente de Pós-graduação); T (Técnico); O (Outros). 
Uma visão detalhada da rede em torno do grupo de autores mais centrais revela algumas informações importantes (Figura 4). O tamanho do nó (e do Id) é proporcional ao número de colaborações (grau do nó) e cada tipo de autor é representado por uma determinada cor.

Figura 4 - Grafo dos autores mais centrais da maior comunidade de autores de publicações da

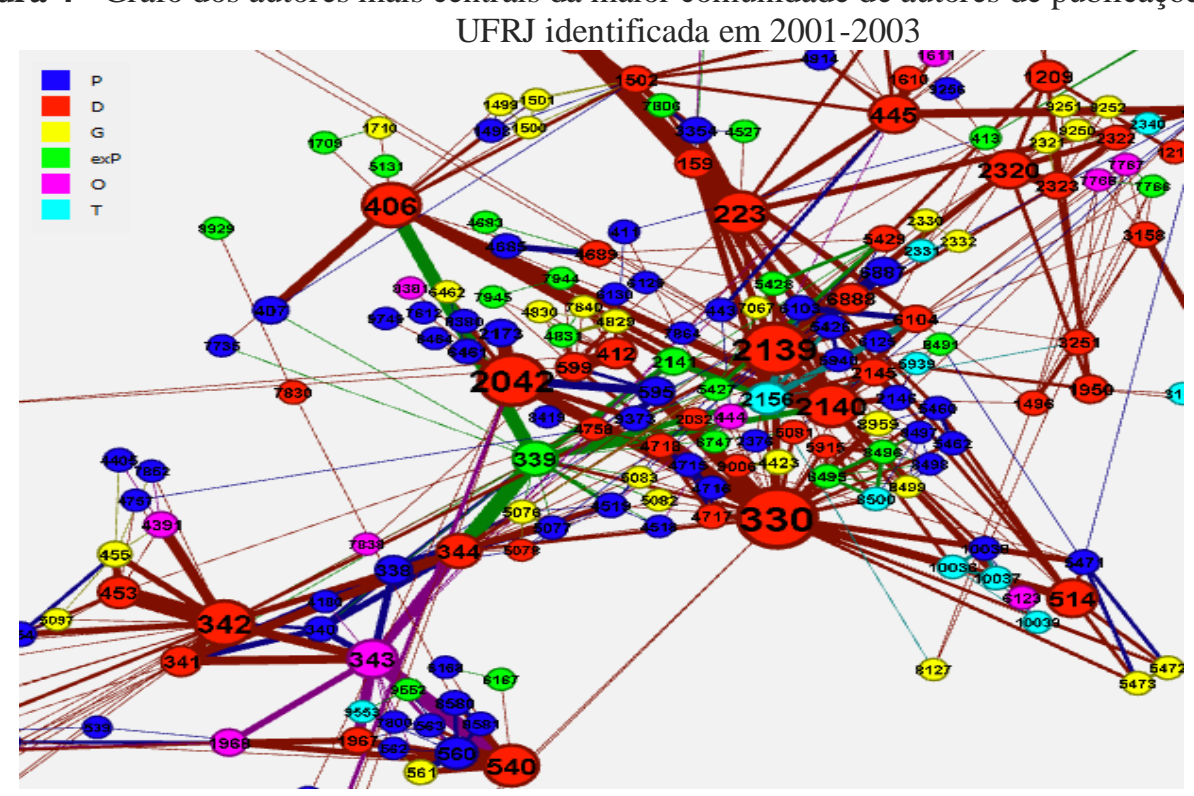

Fonte: Elaborado pelo autor (2018).

Podemos observar claramente que os docentes mais centrais $(\mathrm{id}=330 \mathrm{e}$ 2139) concentram em seu entorno diferentes tipos de autores, formando o principal núcleo de colaboração dessa comunidade. Outros docentes, por sua vez, concentram sua colaboração com algum tipo de autores. Por exemplo, os docentes id= 540 e 2042 colaboram, basicamente, com alunos de pós-graduação $(\mathrm{P})$.

\subsection{Comunidades de autores no triênio 2004-2006}

A rede de autores da UFRJ, no período 2004-2006, é composta por 6.325 autores (total de autores em artigos com colaboração). A identificação de agrupamentos dessa população, aplicando o algoritmo Blondel et al. (2008), identificou um total de 45 comunidades, envolvendo 4.419 autores, os quais mantiveram entre si 14.256 conexões representadas na rede por arestas (Figura $5-\mathrm{A})$. 


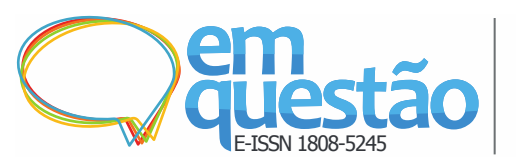

Redes de Interação na Ciência: mapeamento de comunidades de autores

Roberto Mario Lovón Canchumani

Figura 5 - (A) Grafo das comunidades de autores da UFRJ de publicações em periódicos identificadas no período 2004-2006. (B) Grafo da maior comunidade de autores de artigos em periódicos da UFRJ no período 2004-2006

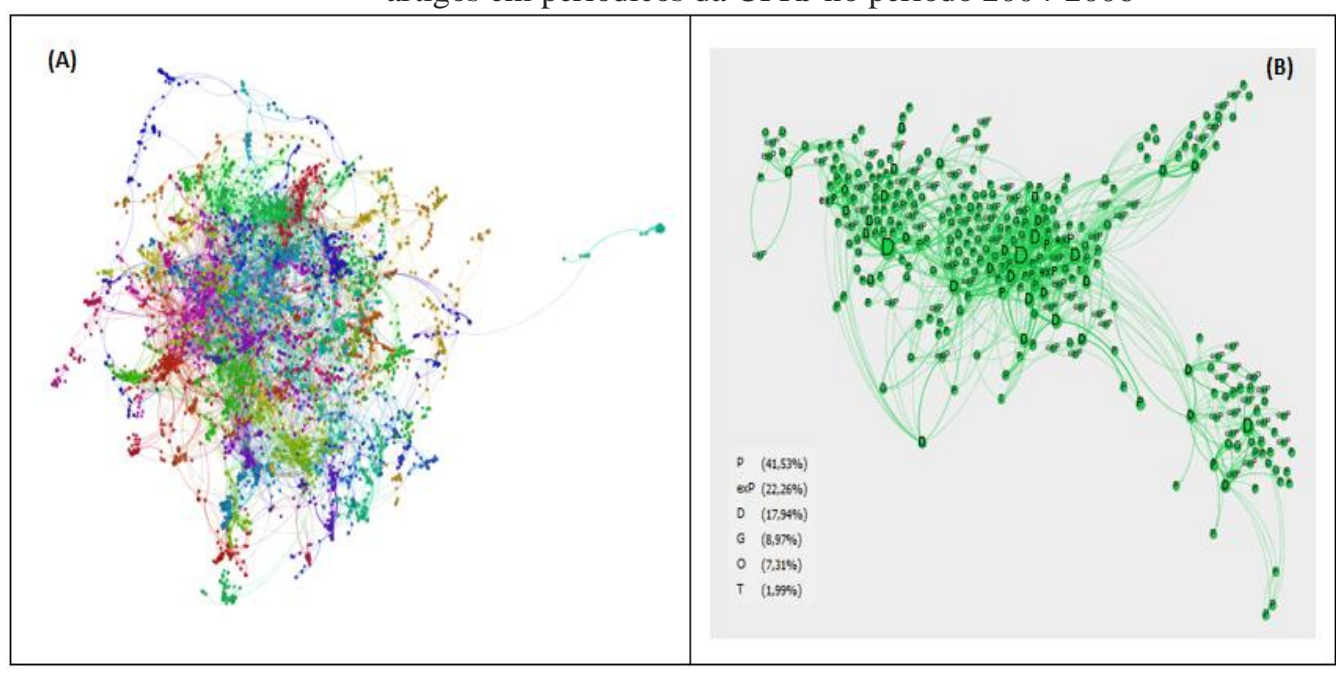

Fonte: Elaborado pelo autor (2018)

O grau médio da rede foi de 6,45 e a transitividade (coeficiente de clustering) de 0,77. Esses dados mostram uma expansão do número de subgrupos (agrupamentos), resultante do maior nível de interação entre autores em relação ao triênio anterior.

A maior comunidade de autores, identificada no período de 2004-2006, é constituída por 301 autores (o que representa 6,81\% do total de autores das 45 comunidades), 65 autores a mais que no triênio anterior. Esses autores estabeleceram 1.127 relacionamentos, arestas (Figura 5-B). O grau médio é de 7,48 , ou seja, cada autor colabora, em média, com mais de sete pesquisadores. $\mathrm{O}$ coeficiente de transitividade apresentado é de 0,80 .

A classificação de autores dessa comunidade por tipo de vínculo mostra que a maioria é composta por alunos de pós-graduação - P (41,53\%), seguida por alunos egressos da pós-graduação - exP $(22,26 \%)$ e docentes - D $(17,94 \%)$. Alunos de graduação $(\mathrm{G})$ representam $8,97 \%$ e autores com outros tipos de vinculo $(\mathrm{O}) 7,31 \%$. Já o número de técnicos $(\mathrm{T})$ participantes nessa comunidade representam $1,99 \%$.

Os 20 autores que apresentam as maiores medidas de centralidade de grau são mostrados na Tabela 2. 
Tabela 2 - Vínculo e grande área dos autores com maiores medidas de centralidade de grau da maior comunidade identificada das publicações da UFRJ em 2004-2006

\begin{tabular}{|c|c|c|c|}
\hline Id & Vinculo* & Grau & Áreas de Atuação \\
\hline 56 & $\mathrm{D}$ & 70 & Ciências Biológicas \\
\hline 233 & $\mathrm{D}$ & 58 & Ciências Biológicas \\
\hline 54 & $\mathrm{D}$ & 45 & Ciências Biológicas \\
\hline 593 & $\mathrm{D}$ & 44 & Ciências Biológicas \\
\hline 55 & $\mathrm{D}$ & 39 & Ciências Biológicas \\
\hline 57 & $\mathrm{D}$ & 38 & Ciências Biológicas \\
\hline 182 & $\mathrm{D}$ & 33 & Ciências Biológicas \\
\hline 600 & $\mathrm{D}$ & 31 & Ciências Biológicas \\
\hline 558 & $\mathrm{D}$ & 30 & Ciências Biológicas \\
\hline 442 & $\mathrm{D}$ & 25 & Ciências Biológicas \\
\hline 3389 & $\mathrm{D}$ & 25 & Ciências da Saúde \\
\hline 53 & exP & 24 & Ciências Biológicas \\
\hline 337 & $\mathrm{D}$ & 24 & Engenharias, Ciências Biológicas \\
\hline 395 & $\mathrm{D}$ & 23 & Ciências Biológicas \\
\hline 244 & $\mathrm{D}$ & 22 & Ciências Biológicas \\
\hline 397 & $D$ & 22 & Ciências Biológicas \\
\hline 833 & $P$ & 21 & Ciências Biológicas \\
\hline 4797 & $D$ & 21 & Ciências da Saúde \\
\hline 1047 & $\mathrm{D}$ & 20 & Ciências Biológicas \\
\hline 6096 & exP & 20 & Ciências Biológicas \\
\hline
\end{tabular}

* Tipos de Vínculo: D (Docente); exP (Egresso Discente de Pós-graduação); P (Discente de Pósgraduação).

Observa-se que dos 20 autores identificados, 17 são docentes, dois são egressos da pós-graduação e um é aluno de pós-graduação.

No tocante às grandes áreas dos autores, verifica-se que 17 deles atuam nas Ciências Biológicas, e dois nas Ciências da Saúde. Apenas um autor atua em duas áreas do conhecimento: Engenharias e Ciências Biológicas.

Uma visão detalhada da rede em torno do grupo de autores mais centrais revela algumas informações importantes (Figura 6). Podemos notar dois grandes polos de colaboração. Um envolvendo os docentes $\mathrm{Id}=56$ e 54, os quais tem fortes ligações com outros docentes e, em seu entorno, alunos e egressos da pósgraduação. 


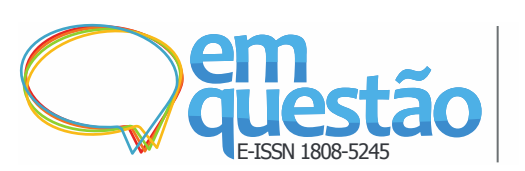

Redes de Interação na Ciência: mapeamento de comunidades de autores

Roberto Mario Lovón Canchumani

Figura 6 - Grafo dos autores mais centrais da maior comunidade de autores de publicações da UFRJ identificada em 2004-2006

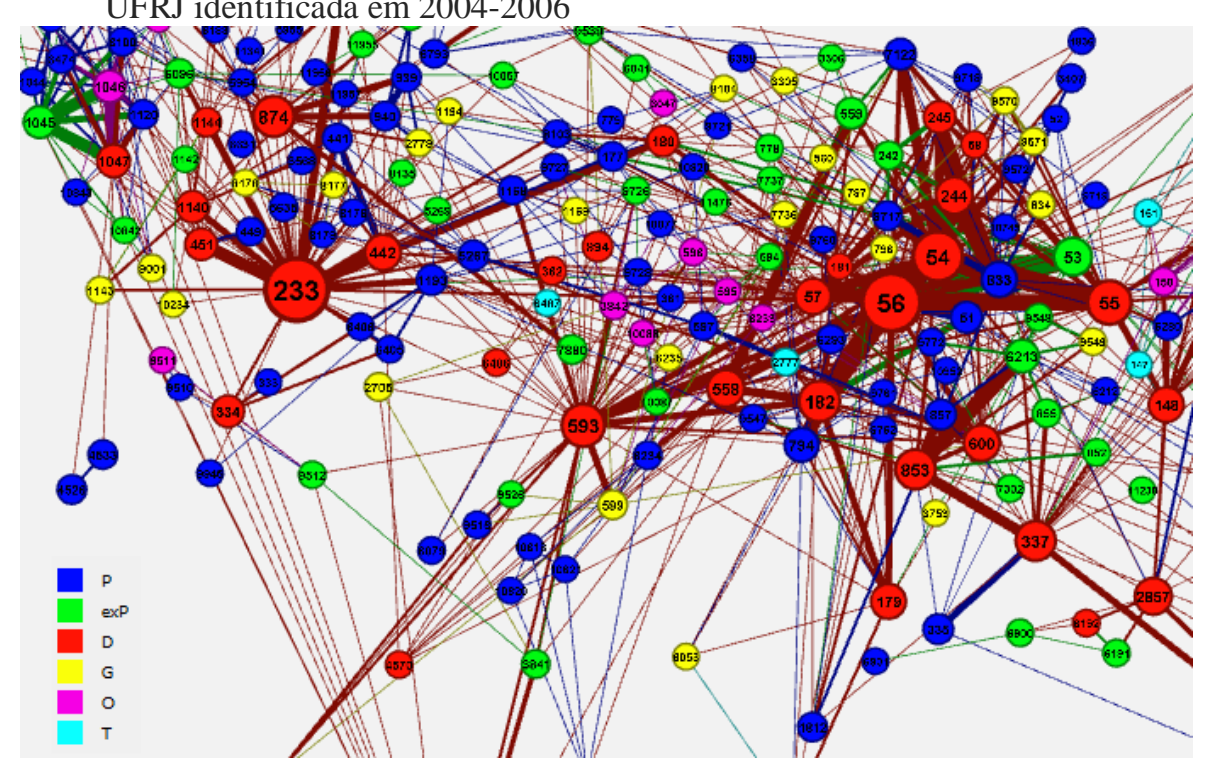

Fonte: Elaborado pelo autor (2018).

Por outro lado, temos o docente $\mathrm{Id}=233$, que concentra sua colaboração com alunos de pós-graduação.

\subsection{Comunidades de autores no triênio 2007-2009}

No período 2007-2009, a rede de autores da UFRJ é composta por 7.484 autores (total de autores em artigos com colaboração).

A identificação de agrupamentos dessa população, aplicando o algoritmo Blondel et al. (2008), identificou um total de 50 comunidades, envolvendo 5.268 autores, os quais mantiveram entre si 17.548 ligações, representadas na rede por arestas (Figura 7-A). O grau médio foi de 7,48 e a transitividade (coeficiente de clustering) de 0,78. Há, portanto, relativo aumento na formação de agrupamentos em relação ao período anterior.

A maior comunidade de autores identificada no período de 2007-2009 é constituída por 301 autores (o que representa 5,71\% do total de autores das 50 comunidades), número similar ao triênio anterior. Esses autores estabeleceram 1.133 relacionamentos, arestas (Figura 7-B). O grau médio é 7,52, isto é, cada autor colabora, em média, com mais de sete pesquisadores, e o coeficiente de clustering é de 0,79 . 


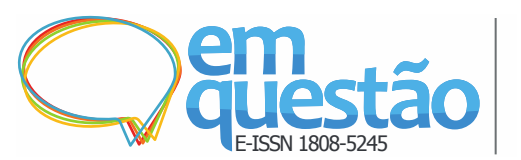

Redes de Interação na Ciência: mapeamento de comunidades de autores

Roberto Mario Lovón Canchumani

Figura 7 - (A) Grafo das comunidades de autores da UFRJ de publicações em periódicos identificadas no período 2007-2009. (B) Grafo da maior comunidade de autores de artigos em periódicos da UFRJ no período 2007-2009

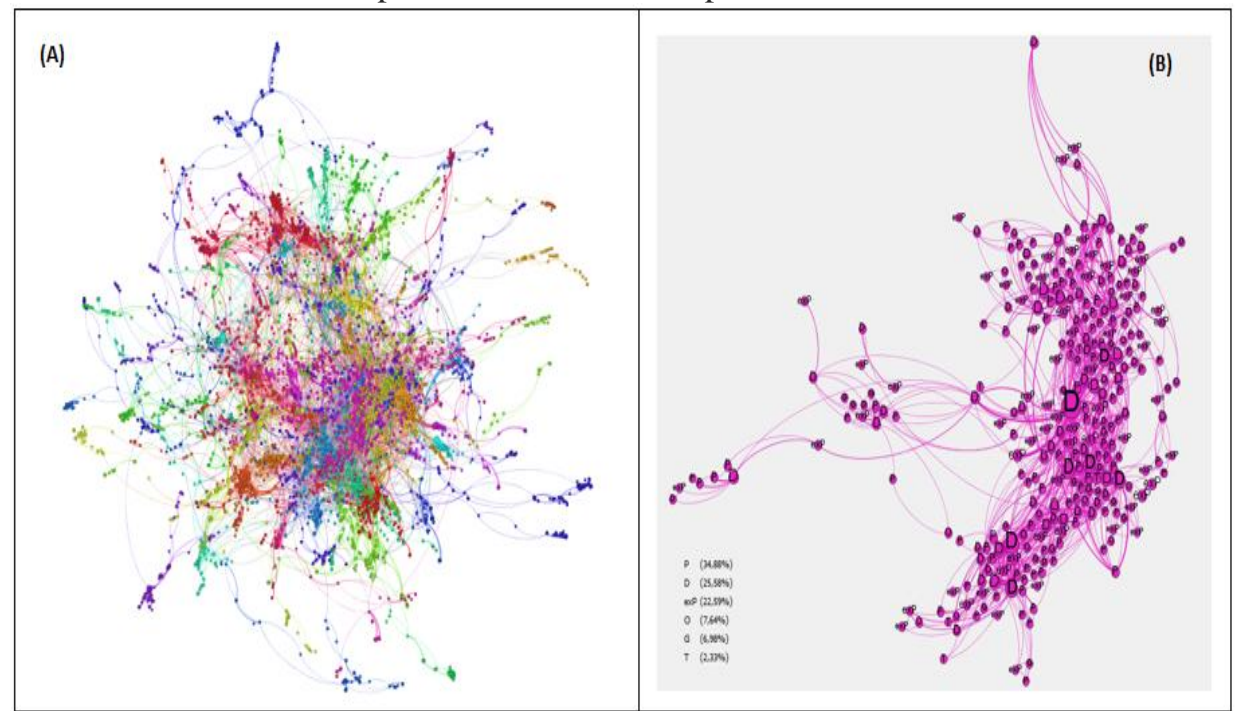

Fonte: Elaborado pelo autor (2018)

A classificação de autores dessa comunidade por tipo de vínculo mostra que a maioria é composta por alunos de pós-graduação - P $(34,88 \%)$, seguida por docentes - D (25,58\%) e egressos da pós-graduação - exP $(22,59 \%)$. Autores com outros tipos de vínculo $(\mathrm{O})$ representam 7,64\%, alunos de graduação $(\mathrm{G})$ $6,98 \%$ e técnicos (T) $2,33 \%$.

Os 20 autores com as maiores medidas de centralidade de grau são mostrados na Tabela 3. Podemos notar que 18 dos autores são docentes, um é aluno de pós-graduação e o outro é egresso da pós-graduação.

Em relação às áreas dos autores, verificamos que todos atuam na área de Ciências Biológicas, sendo que um deles também participa das Ciências Humanas.

Tabela 3 - Vínculo e grande área dos autores com maiores medidas de centralidade de grau da maior comunidade identificada das publicações da UFRJ em 2007-2009

\begin{tabular}{|c|c|c|l|}
\hline Id & Vinculo* $^{*}$ & Grau & Áreas de Atuação \\
\hline 290 & D & 54 & Ciências Biológicas \\
\hline 194 & D & 41 & Ciências Biológicas \\
\hline 1095 & D & 38 & Ciências Biológicas \\
\hline 1365 & D & 34 & Ciências Biológicas \\
\hline 1368 & D & 33 & Ciências Biológicas \\
\hline 193 & exP & 31 & Ciências Biológicas \\
\hline
\end{tabular}




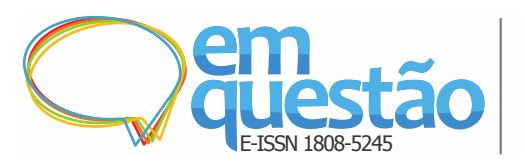

Redes de Interação na Ciência: mapeamento de comunidades de autores

Roberto Mario Lovón Canchumani

\begin{tabular}{|c|c|c|c|}
\hline 1828 & D & 29 & Ciências Biológicas \\
\hline 2078 & D & 29 & Ciências Biológicas \\
\hline 677 & D & 27 & Ciências Biológicas \\
\hline 19 & D & 26 & Ciências Biológicas \\
\hline 293 & D & 26 & Ciências Biológicas \\
\hline 1427 & D & 26 & Ciências Biológicas \\
\hline 294 & D & 25 & Ciências Biológicas \\
\hline 1829 & D & 25 & Ciências Biológicas, Ciências Humanas \\
\hline 3957 & D & 25 & Ciências Biológicas \\
\hline 602 & D & 24 & Ciências Biológicas \\
\hline 1827 & D & 24 & Ciências Biológicas \\
\hline 670 & D & 23 & Ciências Biológicas \\
\hline 1826 & P & 23 & Ciências Biológicas \\
\hline 669 & D & 22 & Ciências Biológicas \\
\hline
\end{tabular}

Fonte: Elaborado pelo autor (2018).

* Tipos de Vínculo: D (Docente); exP (Egresso Discente de Pós-graduação);

$\mathrm{P}$ (Discente de Pós-graduação).

Uma visualização detalhada da rede em torno do grupo de autores mais centrais (Figura 8) mostra maior concentração de colaboração entre docentes. Chama a atenção, por outro lado, a significativa participação, além dos alunos de pós-graduação, dos egressos da pós-graduação em torno dos autores mais representativos em termos de grau $(I d=290$ e 194).

Figura 8 - Grafo dos autores mais centrais da maior comunidade de autores de publicações da

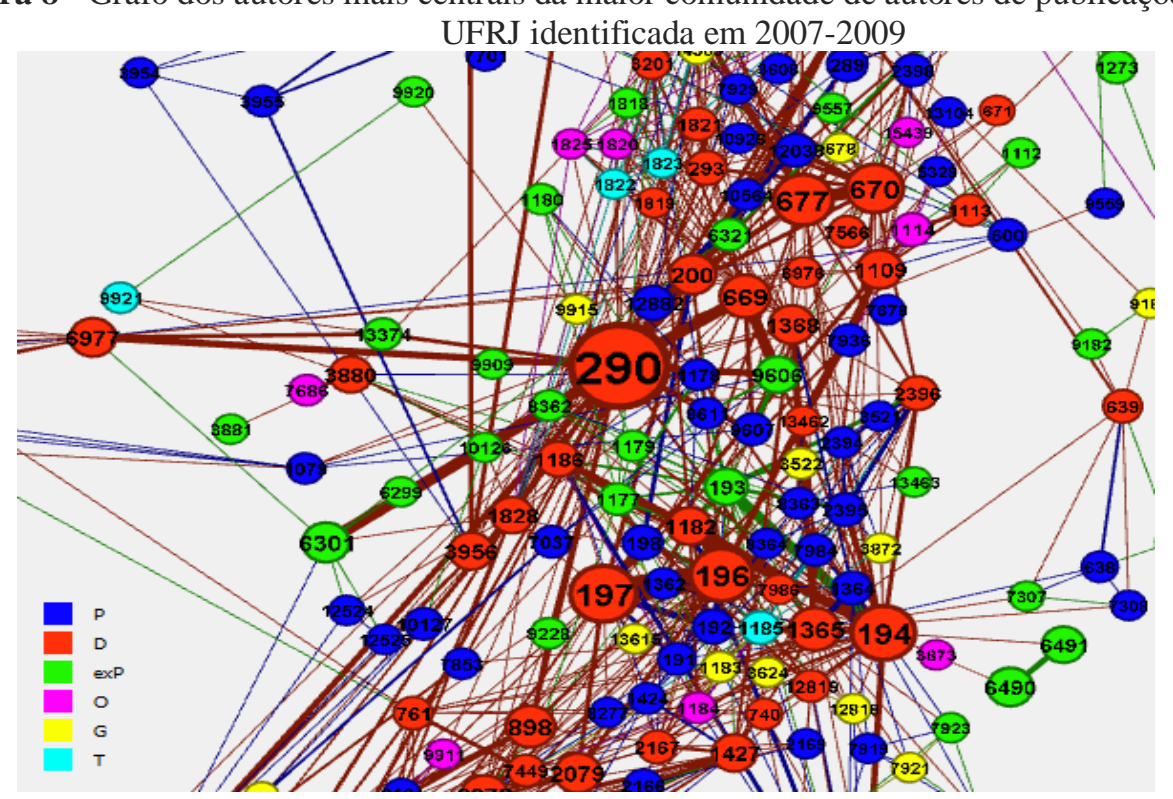

Fonte: Elaborado pelo autor (2018). 


\subsection{Comunidades de autores no triênio 2010-2012}

A rede de autores da UFRJ, no período 2010-2012, é composta por 7.503 autores (total de autores em artigos com colaboração). A identificação de agrupamentos dessa população, aplicando o algoritmo Blondel et al. (2008), apontou um total de 42 comunidades, envolvendo 5.169 autores, os quais mantiveram entre si 19.328 conexões, representadas na rede por arestas (Figura 9-A). O grau médio foi de 7,47 e a transitividade (coeficiente de clustering) de 0,78

Note-se que o número de autores e comunidades diminui em relação ao período anterior, 2007-2009, entretanto, o número de conexões aumentou. Isto pode indicar que o aumento da colaboração ocorreu pelo incremento dos agrupamentos já existentes em períodos anteriores.

Figura 9 - (A) Grafo que mostra as 40 comunidades de autores da UFRJ identificadas no período 2010-2012. (B) Grafo que destaca a maior comunidade de autores da UFRJ identificada no período 2010-2012

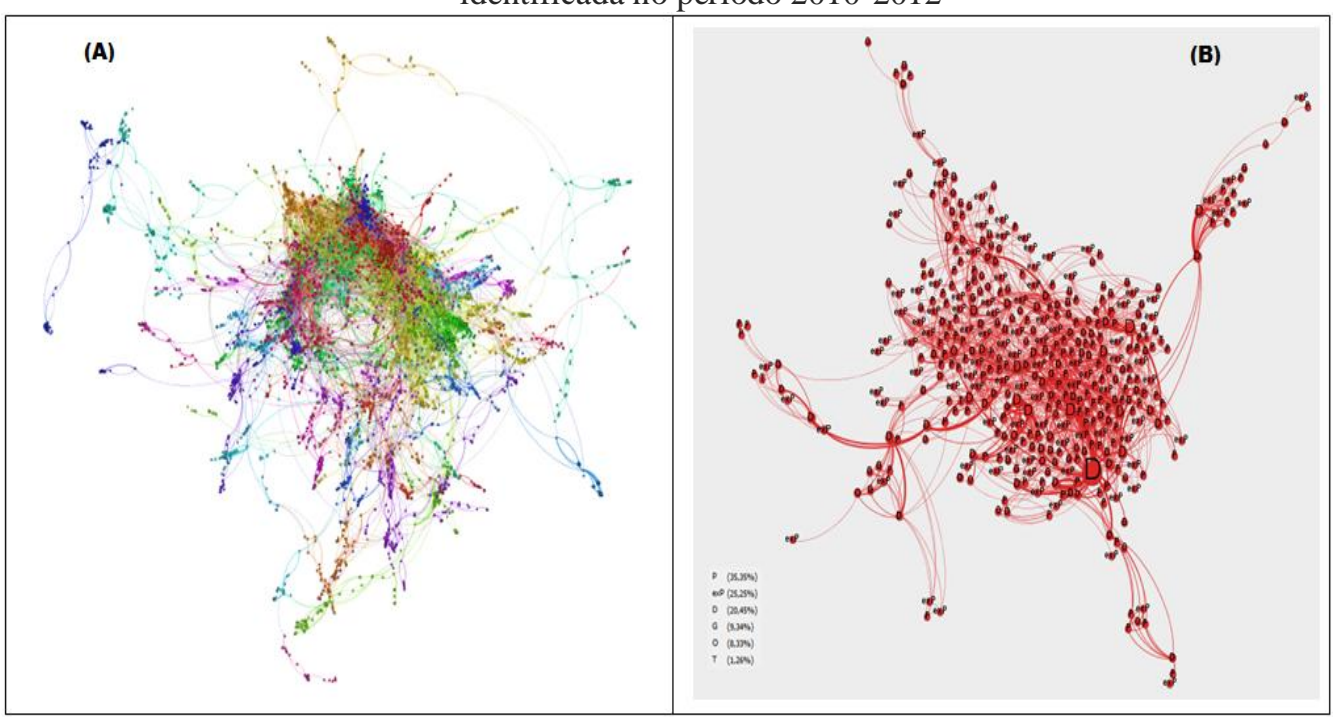

Fonte: Elaborado pelo autor (2018).

A maior comunidade de autores identificada no período de 2010-2012 é constituída por 396 autores (o que representa 7,66\% do total de autores das 40 comunidades), 95 autores a mais que no triênio anterior. Esses autores estabeleceram 1.607 relacionamentos, arestas (Figura 9-B). O grau médio é 
8,12 , isto é, cada autor colabora, em média, com pouco mais de oito pesquisadores, e o coeficiente de clustering é de 0,81 .

Ao verificar os autores dessa comunidade de acordo com seus respectivos tipos de vínculo, vemos que os grupos mais representativos são os alunos de pós-graduação - P (35,35\%), seguido pelos egressos da pós-graduação - exP $(25,25 \%)$ e docentes - D (20,45\%). Os estudantes de graduação (G) representam 9,34\%, os autores com outros tipos de vínculo (O) 8,33\% e os técnicos (T) 1,26\%. Desse total, identificamos os 20 autores que apresentam as maiores medidas de centralidade (Tabela 4).

Tabela 4 - Vínculo e grande área dos autores com maiores medidas de centralidade de grau da maior comunidade identificada das publicações da UFRJ em 2010-2012

\begin{tabular}{|c|c|c|c|}
\hline Id & Vinculo* $^{*}$ Grau & Áreas de Atuação \\
\hline 711 & D & 80 & Ciências Biológicas \\
\hline 536 & D & 47 & Ciências Biológicas \\
\hline 2712 & D & 44 & Ciências Biológicas \\
\hline 180 & D & 43 & Ciências Biológicas \\
\hline 179 & D & 37 & Ciências Biológicas \\
\hline 678 & D & 37 & Ciências Biológicas \\
\hline 350 & D & 36 & Ciências Biológicas \\
\hline 9049 & D & 36 & Ciências Biológicas \\
\hline 167 & D & 34 & Ciências Biológicas \\
\hline 168 & D & 34 & Ciências Biológicas \\
\hline 334 & D & 33 & Ciências Biológicas Biológicas \\
\hline 177 & P & 32 & Ciências Biológicas \\
\hline 1436 & D & 27 & Ciências Biológicas \\
\hline 1623 & D & 27 & Ciências Biológicas \\
\hline 563 & D & 25 & Ciências Biológicas \\
\hline 564 & D & 25 & Ciências Biológicas \\
\hline 1398 & D & 25 & Ciências Biológicas, Ciências Exatas e da Terra \\
\hline 1714 & D & 25 & Ciências Biológicas, Ciências Humanas \\
\hline 1608 & D & 23 & Ciências Biológicas, Ciências da Saúde \\
\hline 1609 & D & 23 & Cich \\
\hline
\end{tabular}

Fonte: Elaborado pelo autor (2018).

* Tipos de Vínculo: D (Docente); P (Discente de Pós-graduação)

Verifica-se que 19 deles são docentes e um aluno de pós-graduação. Em relação às áreas dos autores, constatamos que todos atuam na área de Ciências Biológicas, sendo que três deles atuam também nas seguintes áreas: Ciências da Saúde; Ciências Exatas e da Terra e; Ciências Humanas.

Uma visão detalhada da rede em torno do grupo de autores mais centrais revela algumas informações interessantes (Figura 10). É possível notar forte interação entre os docentes identificados com os números 711 e 179. O primeiro 


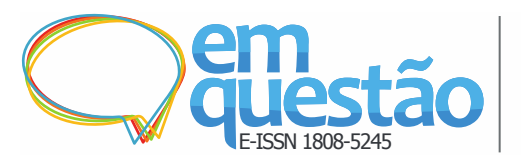

Redes de Interação na Ciência: mapeamento de

comunidades de autores

Roberto Mario Lovón Canchumani

mantendo, em seu entorno, colaborações com outros docentes e, o último, colaborando mais intensamente com alunos de pós-graduação.

Figura 10 - Grafo dos autores mais centrais da maior comunidade de autores de publicações da UFRJ identificada em 2010-2012

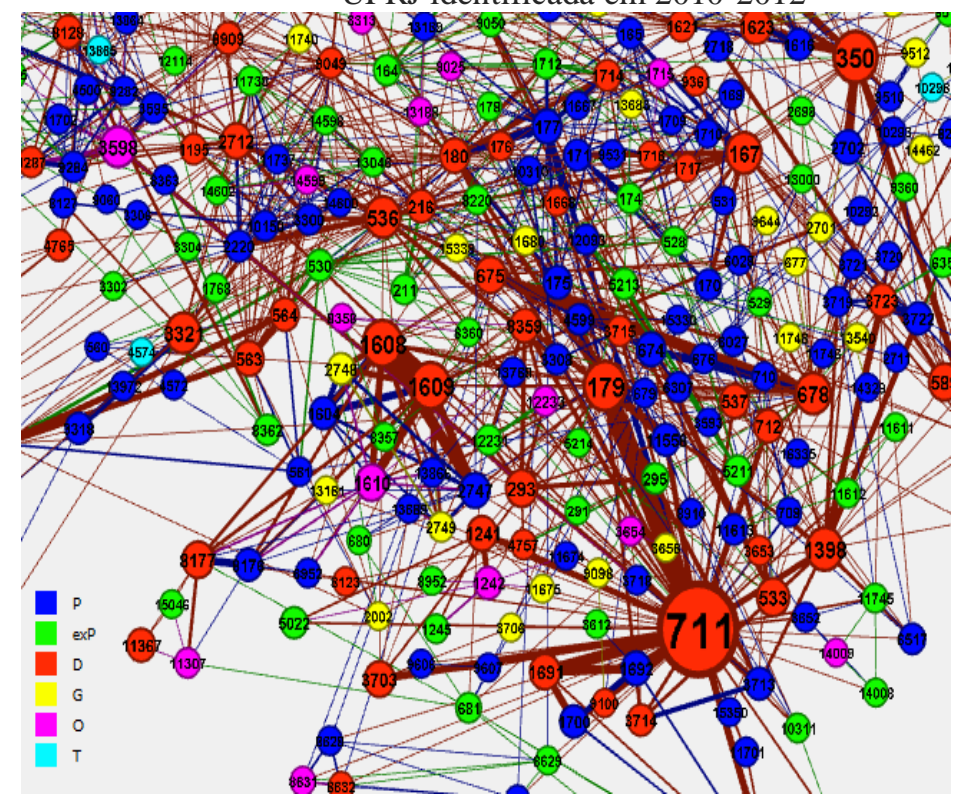

Fonte: Elaborado pelo autor (2018).

\section{Discussão e conclusões}

A maior parte das redes do mundo real caracteriza-se por conter comunidades muito entrelaçadas e ocultas, nas quais seus membros formam subgrupos, configurando uma imbricada rede entre todos eles. A identificação de comunidades possibilita definir e melhor conhecer os subgrupos em função da estrutura de escolha de seus enlaces, isto é, com base na premissa de que os membros de cada subgrupo tendem a escolher os mesmos colaboradores e serem escolhidos pelos mesmos autores. Dessa forma, a permanência em um subgrupo estabelece-se em função das similaridades de escolha, dadas e recebidas, por cada autor.

Nesse sentido, as métricas de grau e de transitividade mostraram que, ao longo do período analisado, novas colaborações entre autores da UFRJ foram estabelecidas, configurando novos agrupamentos/comunidades, tornando, dessa maneira, maior a transitividade da rede. Em outras palavras, ao logo dos triênios, a tendência dos autores da UFRJ se agruparem aumenta. 


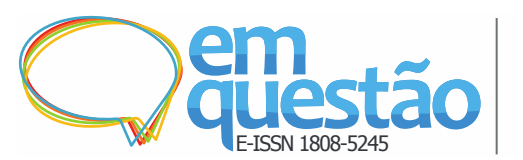

Redes de Interação na Ciência: mapeamento de

Assim, no período de 2001-2003, se identificou um total de 40 comunidades de autores, envolvendo 3.736 autores. Em média, esses autores participaram de publicações com pouco mais de seis autores (o grau médio foi de 6,05), e a transitividade foi de 0,76. Ao analisar a maior comunidade identificada nesse período, se verificou que ela é composta por 236 autores, e que cada autor colabora, em média, com cinco pesquisadores. O coeficiente de transitividade apresentado foi de 0,79. No período 2004-2006, houve um aumento do nível de colaboração e expansão do número de agrupamentos/comunidades $(n=45)$ em relação ao período anterior. No triênio seguinte (2007-2009), também se observou aumento do nível de colaboração e formação de agrupamentos/comunidades $(n=50)$ em relação ao período anterior.

No último triênio, por outro lado, se identificou um total de 42 comunidades, envolvendo 5.169 autores (o grau médio foi de 7,47, e a transitividade foi de 0,78), ou seja, diminuiu o número de autores e de comunidades em relação ao período anterior. Isto indica que houve uma intensificação das interações nos agrupamentos já existentes em períodos anteriores. Ao analisar a maior comunidade identificada nesse período (20102012), verificou-se que ela é composta por 396 autores, 95 autores a mais que no triênio anterior, e que cada autor colabora, em média, com pouco mais de oito pesquisadores (grau médio 8,12). A transitividade apresentada foi de 0,81 .

Este quadro constitui traço característico da ciência contemporânea, sendo cada vez mais uma atividade coletiva, multidisciplinar e na qual participam diversos atores. A complexidade cada vez maior dos problemas de pesquisa supõe que pesquisadores isolados são capazes de tratar uma quantidade limitada de informações. Assim sendo, pesquisadores procuram se agrupar e interagir através de colégios invisíveis (SOLLA PRICE, 1965; CALLON et al., 1995).

Observar, portanto, o desempenho científico do corpo social de uma instituição é de particular interesse para obter informações sobre o grau de eficiência e envolvimento dos diferentes recursos humanos na ciência. Muitos estudos têm buscado esse relacionamento por meio de diferentes abordagens. Larivière (2012), por exemplo, investigou o desempenho de todos os alunos de 
doutorado matriculados em cursos de pós-graduação em universidades de Quebec, que, segundo o autor, é o primeiro estudo em grande escala com esse foco. Entre os resultados, o autor destaca a alta contribuição de estudantes de doutorado: participando da autoria de cerca de 30\% dos artigos de toda a província.

Em nosso caso, envolvendo a UFRJ, o foco não foi apenas no desempenho dos alunos de doutorado, mas em todos os tipos de relações institucionais. Dessa forma, ao observar a maior comunidade de autores identificada em 2001-2003, se verificou que a maioria de autores é composta por alunos de pós-graduação $(38,02 \%)$, seguida por docentes $(25,48 \%)$ e alunos de graduação (12,93\%). Alunos egressos da pós-graduação representam 9,89\% e os autores com outros tipos de vínculo 8,75\%. Já os técnicos representam 4,94\%.

No último triênio observado (2010-2012), a classificação de autores da maior comunidade identificada nesse período mostrou que a maioria é composta por alunos de pós-graduação $(35,35 \%)$, seguido pelos ex-alunos de pósgraduação $(25,25 \%)$ e docentes $(20,45 \%)$. Os estudantes de graduação representam 9,34\%, os autores com outros tipos de vínculo $8,33 \%$ e os técnicos $1,26 \%$.

Esta análise só foi possível graças à principal fonte de informação deste trabalho, o SIGMA.UFRJ. Trata-se, portanto, de um estudo singular, de ampla abrangência, uma vez que envolve todo o corpo social de uma das principais instituições de ensino e pesquisa do Brasil.

Por fim, cabe ressaltar que uma das contribuições destacáveis deste estudo é a particular visão que proporciona sobre a produção científica da UFRJ, já que, a diferença de outros estudos bibliométricos, a abordagem conduzida não se restringe às limitações de realizar estudos deste tipo utilizando as tradicionais bases de dados internacionais - Web of Science (WoS) ou Scopus. Bases como essas não permitem observar determinadas especificações da produção científica como, por exemplo, o tipo de vínculo institucional dos autores como aqui mostrado. 


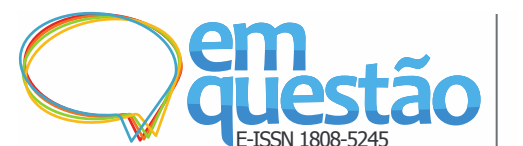

Redes de Interação na Ciência: mapeamento de comunidades de autores

Roberto Mario Lovón Canchumani

\section{Financiamento}

Este trabalho contou com financiamento da Coordenação de Aperfeiçoamento de Pessoal de Nível Superior (CAPES).

\section{Referências}

BARABÁSI, A.; JEONG, H; NÉDA, Z.; RAVASZ, E; SCHUBERT, A; VICSEK, T. Evolution of the social network of scientific collaborations. Physica A, v. 311, n. 3, p. 590- 614, 2002.

BARBOSA, Diego Andrés de Barros Lima.; AVELINO, Leonardo Borges; SOUZA, Rarylson Freitas de.; OLIVEIRA, Camila Cristina Gomes Ferreira de; JUSTEL, Claudia. Medidas de centralidade e detecção de comunidades em rede de co-autoria. In: SIMPÓSIO BRASILEIRO DE PESQUISA OPERACIONAL, 43., 2011, Ubatuba. Anais... Ubatuba, 2011. p. 1-10.

BLONDEL, Vincent D.; GUILLAUME, Jean-Loup; LAMBIOTTE, Renaud; LEFEBVRE, Etienne. Fast unfolding of communities in large networks. J. Stat. Mech, p.100-108, 2008.

CALLON, Michel; COURTIAL, Jean-Pierre; PENAN, Hérve. Cienciometría. El estudio cuantitativo de la actividad científica: de la bibliometría a la vigilancia tecnología. Gijón: Trea, 1995.

CANCHUMANI, Roberto Mario Lóvon. Redes de Interação Científica: identificação de comunidades de autores na UFRJ. In: ENCONTRO BRASILEIRO DE BIBLIOMETRIA E CIENTOMETRIA, 6., 2018, Rio de Janeiro. Anais.... Rio de Janeiro: UFRJ, 2018. p. 333-343.

CANCHUMANI, Roberto Mario Lóvon; LETA, Jacqueline; FIGUEIREDO, Antonio MacDowell de. Domínios Científicos: mapeamento de áreas do conhecimento da Universidade Federal do Rio de Janeiro. Inf. \& Soc: Est, v. 27, n. 2, p.199-218, 2017.

FORTUNATO, Santo. Community detection in graphs. Physics Reports, v. 486, p. 75-174, 2010.

LARIVIERE, Vincent. On the shoulders of students? The contribution of $\mathrm{PhD}$ students to the advancement of knowledge. Scientometrics, v. 90, n. 2, p. 463- 481, 2012.

PALLA, Gergely; DERÉNYI, Imre; FARKAS, Illes; VICSEK, Tamas. Uncovering the overlapping community structure of complex networks in nature and society. Nature, 435, p. 814- 818, 2005. 
RIVELLINI, Giulia; RIZZI, Ester; ZACCARIN, Susanna. The science network in Italian population research: an analysis according to the social network perspective. Scientometrics, v. 67, n. 3, p. 407-418, 2006.

PERIANES-RODRIGUEZ, Antonio; OLMEDA-GÓMEZ, Carlos; MOYAANEGON, Félix. Detecting, identifying and visualizing research groups in coauthorship networks. Scientometrics, v. 82, n. 2, p. 307-319, 2010.

RODRIGUEZ, Josep A. Análisis estructural y de redes. Madrid: Centro de Investigaciones Sociológicas, 1995.

SOLLA PRICE, Derek J. Networks of scientific papers. Science, v. 149, n. 3.683, p. 510-515, 1965.

\title{
Scientific Interaction Networks: mapping of author communities
}

\begin{abstract}
It provides the results of a proposal of identification of authors communities realized at the Federal University of Rio de Janeiro - UFRJ. The objective was to identify the clustering tendency of authors of the institution over time, as well as to observe more specific characteristics of the main communities identified. The study investigated 44,233 records retrieved from the institutional database, SIGMA.UFRJ, articles published in journals from 2001 to 2012. Data were organized and analyzed using Excel and Gephi software. The metrics of degree and transitivity/modularity showed that, over the study period, new collaborations among authors of UFRJ were established, forming new clusters/communities of authors. The largest authors' clusters/communities were detailed and revealed their main specifications
\end{abstract}

Keywords: Authors Communities. Collaboration Networks. Co-authorship.

Recebido: 23/09/2017

Aceito: 03/12/2018 